\title{
In Silico Screening of Potential Chinese Herbal Medicine Against COVID-19 by Targeting SARS-CoV-2 3CLpro and Angiotensin Converting Enzyme II Using Molecular Docking*
}

\author{
GAO Liang-qin ${ }^{1}, \mathrm{XU}$ Jing $^{2}$, and CHEN Shao-dong ${ }^{3}$
}

\begin{abstract}
Objective: To seek potential Chinese herbal medicine (CHM) for the treatment of coronavirus disease 2019 (COVID-19) through the molecular docking of the medicine with SARS-CoV-2 3CL hydrolytic enzyme and the angiotensin converting enzyme II (ACE2) as receptors, using computer virtual screening technique, so as to provide a basis for combination forecasting. Methods: The molecular docking of CHM with the SARS-Cov-2 3CL hydrolase and the ACE2 converting enzyme, which were taken as the targets, was achieved by the Autodock Vina software. The CHM monomers acting on 3CLpro and ACE2 receptors were retrieved from the Traditional Chinese Medicine Systems Pharmacology Database and Analysis Platform, the active ingredients were selected, and the key $\mathrm{CHMs}$ and compounds were speculated. Based on the perspective of network pharmacology, the chemical-target network was constructed, and the functional enrichment analysis of gene ontology and the pathway enrichment analysis of Kyoto encyclopedia of genes and genomes were carried out by DAVID to speculate about the mechanism of action of the core drug pairs. Results: There are 6 small molecule compounds that have the optimal binding energy with the two target proteins. Among 238 potential anti-COVID-19 herbs screened in total, 16 kinds of CHM containing the most active ingredients, and 5 candidate anti-COVID-19 herbs that had been used in high frequency, as well as a core drug pair, namely, Forsythiae Fructus-Lonicerae Japonicae Flos were selected. Conclusion: The core drug pair of Forsythiae Fructus-Lonicerae Japonicae Flos containing multiple components and targets is easy to combine with $3 C$ Lpro and ACE2, and exerts an anti-COVID-19 pneumonia effect through multi-component and multi-target, and plays the role of anti-COVID-19 pneumonia in multi-pathway.
\end{abstract}

KEYWORDS molecular docking, COVID-19, SARS-CoV-2 3CL hydrolytic enzyme, angiotensin converting enzyme II , Chinese medicine

COVID-19 caused by the new coronavirus is characterized by rapid and extensive spread, strong infectivity and general susceptibility of the population. Currently, there is no specific drug for it. ${ }^{(1)}$ On February 11, 2020, the International Commission on the Classification of Viruses announced the official designation of the new coronavirus as severe acute respiratory syndrome coronavirus 2 (SARS-CoV-2, formerly known as 2019-ncov). On the same day, the World Health Organization (WHO) named the disease caused by this virus as Corona Virus Disease 2019 (COVID-19). Coronaviruses (CoV) are a large family of viruses that cause illness ranging from the common cold to more severe diseases such as Middle East Respiratory Syndrome and Severe Acute Respiratory Syndrome. The new coronavirus isolated in Wuhan is found to be the 7th corona virus that can infect humans. ${ }^{(2)}$ The s-protein expressed by the cov-2 virus binds to the angiotensin-converting enzyme II (ACE2) in the human body, thereby infecting cells, invading the body and causing diseases. ${ }^{(3)}$ Recently, the team of Rao ZH and Yang HT from Shanghai University of Science and Technology has obtained a high-

CThe Chinese Journal of Integrated Traditional and Western Medicine Press and Springer-Verlag GmbH Germany, part of Springer Nature 2020

*Supported by the Fundamental Research Funds for the Central Central University, Scientific Research Project on Prevention and Treatment Emergency in Novel Coronavirus (No.20720200034)

1. Department of Traditional Chinese Medicine, Xiamen University Hospital, Xiamen University, Xiamen, Fujian Province (361005), China; 2. College of Ocean and Earth Sciences, Xiamen University, Xiamen, Fujian Province (361102), China; 3. Department of Traditional Chinese Medicine, School of Medicine, Xiamen University, Xiamen, Fujian Province (361002), China

Corresponding to Prof. CHEN Shao-dong, E-mail:adong@xmu. edu.cn

DOI: https://doi.org/10.1007/s11655-020-3476-x 
resolution crystal structure of 2019-nCoV coronavirus $3 \mathrm{CL}$ hydrolase (Mpro) which is currently considered to be an effective target of the COVID-19 virus. ${ }^{(4)}$ These studies have brought hope for seeking effective clinical drugs to prevent and control COVID-19, which may help us to develop more effective ways to fight against COVID-19.

COVID-19 belongs to the category of "epidemic" disease of Chinese medicine (CM). Since CM has played an active role through integration of Chinese and Western medicine in the treatment of COVID-19, its clinical efficacy has been widely praised. The study of Wang, et $\mathrm{al}^{(5)}$ showed that the cure rate of patients with new corona virus pneumonia might be positively correlated with the participation rate of $\mathrm{CM}$ treatment. In the "Diagnosis and Treatment Program of Novel Coronavirus Pneumonia (3rd trial edition)", ${ }^{(1)}$ the specific methods of CM prevention and control were identified, and were constantly supplemented and improved in the 4 th to 7 th editions, ${ }^{(1)}$ playing an important role in the prevention and control of COVID-19. ${ }^{(1)}$ Due to the holistic "multi-target, multipathway and multi-effect" therapeutic characteristics, single herbs and CHM compounds are advantageous and potential in the treatment of some diseases.

Molecular docking is a method of drug design based on the characteristics of the receptor and the way the receptor interacts with the drug molecule. As an emerging research method combining the physical and chemical principles and with the scientific calculation algorithm, it provides a feasible strategy for exploring the basis and mechanism of the pharmacodynamic substances of $\mathrm{CHM}$, and promotes the modern research process of $\mathrm{CHM}{ }^{(6)}$

This study took the SARS-CoV-2 3CL hydrolytic enzyme and the ACE2 as receptors, and molecular docking of the two was performed to select potential antiviral active ingredients, so as to provide reference for seeking effective and quick-acting anti-COVID-19 chemical components.

\section{METHODS}

\section{Database and Software}

Data used in this study were downloaded from the database system: Traditional Chinese Medicine Systems Pharmacology Database and Analysis Platform (TCMSP, http://tcmspw.com/tcmsp.php), Protein Data
Bank (PDB, https://www.rcsb.org/), the Swiss Target Prediction (http://www.swisstargetprediction.ch/), the STRING Online Database (https://string-db.org/), and the Biological Information Annotation Database DAVID, (https://david.ncifcrf.gov/summary.jsp, Version 6.8). The software used included AutoDockTools1.5.6 Software, AutoDockVina Software (http://vina.scripps.edu/), biological information analysis tools Cytoscape v3.8.1, data analysis tools $R$ 3.6.2, protein molecules and visualization software PyMOL.

\section{Molecular Docking}

A total of 12,541 compounds in MOL2 format were downloaded from the TCMSP database, and $3 D$ structures of them were downloaded from PDB database, to establish a virtual screening small molecule database for molecular docking technology. The 3D structure of ACE2 (PDB ID: 1R42) protein (in PDB format) was downloaded from PDB database (https://www.rcsb.org/). SARS-CoV-2 is determined to be a high-resolution crystal structure (PDB ID: 6LU7) of SARS-CoV-2 3CL hydrolase (Mpro) by Rao ZH and Yang HT's research group from Shanghai University of Science and Technology. Visualization and analysis of protein-ligand complexes was performed using PyMOL version 0.99 Software (De Lano Scientific LLC, CA, USA) and high-throughput molecular docking was carried out by Autodock Vina and R. According to the optimal binding energy (affinity) of each compound with the 3CL hydrolase and ACE2 converting enzyme, a small molecule database of anti-COVID-19 CM was established.

\section{Based on ADME Assessment}

"ADME" refers to the absorption, distribution, metabolism and excretion process of exogenous chemicals in the body, and screening ADME properties is an important means to further develop candidate drugs. ${ }^{(7)}$ In this study, the active ingredients that met the drug screening criteria [oral availability $(O B) \geqslant 30 \%$ and drug-likeness $(D L) \geqslant 0.18$ ] in the small-molecule database were selected, so as to find compounds with good pharmacokinetic properties and bioavailability, and to improve the screening efficiency.

\section{Screening of Anti-COVID-19 CHM}

Based on the above screening results, we speculated the anti-COVID-19 novel corona virus pneumonia $\mathrm{CHM}$. We selected $\mathrm{CHM}$ varieties frequently used in prevention and treatment of COVID-19 issued 
by the country and various institutions, and predicted the antiviral core drug pair according to the distribution of active ingredients in these $\mathrm{CHM}$ varieties.

\section{Prediction of Molecular Mechanism of Core Drug Pair Based on Network Pharmacology}

The main active components of the herbs were screened by TCMSP extraction, and the potential targets were predicted by the Swiss Target Prediction server. The STRING database was used to analyze the relationship between drug targets, and the visual analysis was conducted with the help of the Cytoscape 3.5.1 software. Then, the potential targets of the selected active components were submitted to the bioinformatics database DAVID 6.8 for functional annotation of Gene Ontology (GO) genes and enrichment analysis of the Kyoto encyclopedia of genes and genomes (KEGG) pathway in order to further understand the functions of the targets and their role in the signaling pathway, thereby exploring and predicting the potential molecular mechanism of the core drug pair.

\section{RESULTS}

\section{Anti-COVID-19 Molecule Database}

In order to minimize the probability of false positive results, the optimal binding energy of small molecule compounds in $\mathrm{CHM}$ was compared with that of the currently recommended clinical chemical drugs in this study, and the binding energy in the screening criteria was changed to $\leqslant-5.0 \mathrm{kCal} / \mathrm{mol}(-20 \mathrm{~kJ} / \mathrm{mol})$. The partial results are shown in Table 1.

The binding energy of the molecular docking between puerarin and the two target proteins was lower than that of the molecular docking between clinical recommended chemical drugs, and the same two proteins, which indicated that the binding activity of puerarin and target proteins was stronge, which came from Radix Bupleuri, Cyathulae Radix, Puerariae Flos, Hemerocallis Radix and Radix Puerariae. Bicuculline is derived from Corydalis Bungeanae Herba, Forsythiae Fructus, Corydalis Decumbens (Thunb.) Pers, and Corydalis Rhizoma, luteolin is originated from Lonicerae Japonicae Flos, Forsythiae Fructus, Ephedra Herba and other 89 herbs.

\section{Anti-COVID-19 Core Drug Pairs}

The binding activity of 6 active ingredients with the two target proteins were better than that of the
Table 1. Binding Energy of Representative Components and Clinically Recommended Chemical Drugs with SARS-CoV-2 3CL and ACE2

\begin{tabular}{llccc}
\hline Compound & Formula & $\mathrm{MW}$ & $\begin{array}{c}\text { 3CLpro } \\
(\mathrm{kJ} / \mathrm{mol})\end{array}$ & $\begin{array}{c}\mathrm{ACE} 2 \\
(\mathrm{~kJ} / \mathrm{mol})\end{array}$ \\
\hline Puerarin & $\mathrm{C}_{21} \mathrm{H}_{20} \mathrm{O}_{10}$ & 432.38 & -33.47 & -38.07 \\
Bicuculline & $\mathrm{C}_{20} \mathrm{H}_{17} \mathrm{NO}_{6}$ & 367.40 & -26.78 & -41.42 \\
Luteolin & $\mathrm{C}_{15} \mathrm{H}_{10} \mathrm{O}_{6}$ & 286.24 & -26.78 & -36.82 \\
Quercetin & $\mathrm{C}_{15} \mathrm{H}_{10} \mathrm{O}_{7}$ & 302.24 & -26.36 & -36.40 \\
Isorhamnetin & $\mathrm{C}_{16} \mathrm{H}_{12} \mathrm{O}_{7}$ & 316.27 & -25.95 & -35.15 \\
Irisolidone & $\mathrm{C}_{17} \mathrm{H}_{14} \mathrm{O}_{6}$ & 314.29 & -25.53 & -38.49 \\
Lopinavir & $\mathrm{C}_{37} \mathrm{H}_{48} \mathrm{~N}_{4} \mathrm{O}_{5}$ & 628.80 & -22.59 & -37.24 \\
Ritonavir & $\mathrm{C}_{37} \mathrm{H}_{48} \mathrm{~N}_{6} \mathrm{O}_{5} \mathrm{~S}_{2}$ & 720.94 & -24.69 & -36.40 \\
Remdesivir & $\mathrm{C}_{27} \mathrm{H}_{35} \mathrm{~N}_{6} \mathrm{O}_{8} \mathrm{P}$ & 602.58 & -25.94 & -36.40 \\
Arbidol & $\mathrm{C}_{22} \mathrm{H}_{25} \mathrm{BrN}_{2} \mathrm{O}_{3} \mathrm{~S}$ & 531.89 & -28.03 & -30.54 \\
Chloroquine & $\mathrm{C}_{18} \mathrm{H}_{26} \mathrm{ClN}_{3}$ & 319.87 & -24.30 & -27.20 \\
Ribavirin & $\mathrm{C}_{37} \mathrm{H}_{48} \mathrm{~N}_{6} \mathrm{O}_{5} \mathrm{~S}_{2}$ & 720.96 & -25.52 & -32.22 \\
Nitazoxanide & $\mathrm{C}_{12} \mathrm{H}_{9} \mathrm{~N}_{3} \mathrm{O}_{5} \mathrm{~S}$ & 307.28 & -23.85 & -34.73 \\
\hline
\end{tabular}

recommended chemical drugs with these two targets, and a total of 238 potential anti-COVID-19 CHM were chosen, and $42.02 \%$ of them possess the character of Fei (Lung) meridian tropism. Among the potential $\mathrm{CHM}$, heat-clearing herbs accounted for the largest proportion $(29.41 \%)$, followed by tonic herbs $(9.66 \%$, Figure 1). Based on the CM prevention and treatment plans issued by the municipal, provincial and national health administration departments, 5 kinds of frequently used $\mathrm{CHMs}$ containing the most active ingredients from 16 herbs (Figure 2). Among these selected CHMs, Forsythiae Fructus and Lonicerae Japonicae Flos are the most widely distributed active ingredients, and thus selected as the predicted core drug pair in this study.

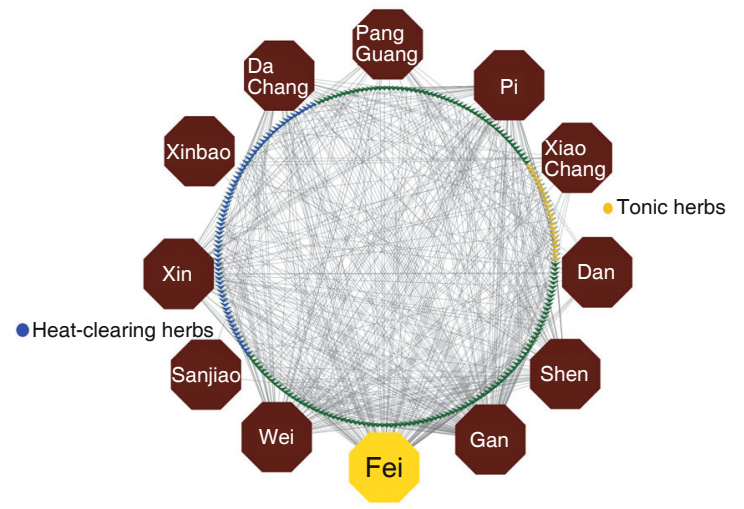

Figure 1. Main Categories of Selected CHMs

GO Enrichment and KEGG Pathway Annotation Analysis of Potential Targets of Forsythiae Fructus-Lonicerae Japonicae Flos

Forsythiae Fructus-Lonicerae Japonicae 


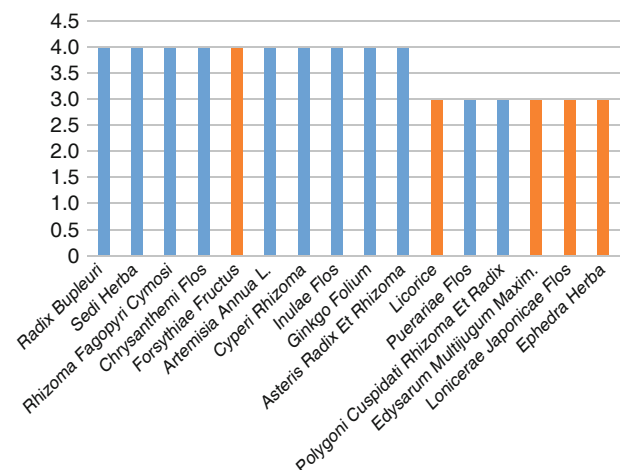

Figure 2. CHMs with Active Ingredient Distribution $\geqslant 3$

Flos contains 46 active ingredients with good pharmacokinetic properties and bioavailability, with a total of 412 potential targets, 121 of which are targeted for viral pneumonia (Figure 3).

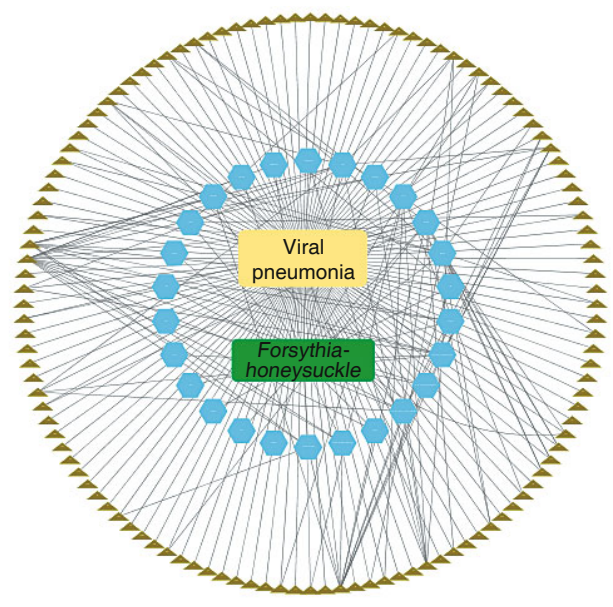

Figure 3. Molecule-Target-Pathway Network of Forsythiae Fructus-Lonicerae Japonicae Flos

Potential targets were imported into the DAVID database for GO term and KEGG pathway enrichment analyses. GO term enrichment results were divided into biological process (BP), cell compound (CC), and molecular function (MF), and showed $35 \mathrm{BP}, 4 \mathrm{CC}$ and $18 \mathrm{MF}$ enriched for these targets had a $P$ value less than 0.05. In addition, 56 KEGG pathways were recognized as $P<0.05$, among which 14 were coincide with the super pathway of viral pneumonia (Figure 4). Pertussis, tuberculosis, T cell receptor (TCR) and the tumor necrosis factor (TNF) signaling pathways had the smallest $P$ values and the most enriched targets.

\section{DISCUSSION}

The COVID-19 belongs to the category of pestilent disease in CM. As mentioned in Detailed Analysis of Warm Diseases (Wen Bing Tiao Bian), a book on epidemic febrile disease written by WU

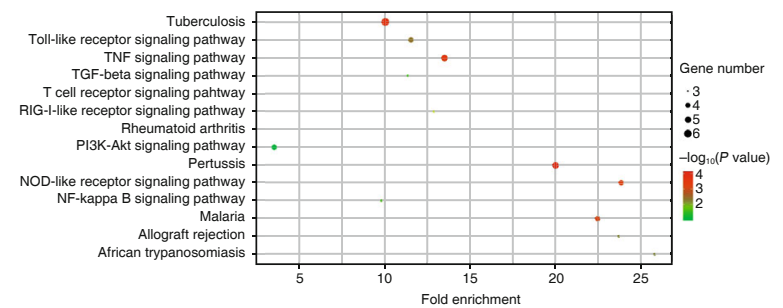

Figure 4. Enrichment Analysis of Potential Target KEGG Pathway of Forsythiae Fructus-Lonicerae Japonicae Flos

Ju-tong, "the pestilence is severe and prevalent." That exactly describes the strong infectivity of the new corona virus pneumonia as a kind of "Wenyi (pestilence) disease". YE Tian-shi in his Treatise on Warm-Heat Disease (Wen Re Lun) stated that "Lung (Fei) is the first to be attacked when a person suffers from warm-heat evils." In this study, a total of 238 potential anti-COVID-19 CHMs were selected based on the analysis of 2 target proteins SARS-CoV-2 $3 \mathrm{CL}$ hydrolytic enzyme and ACE2. As a pestilence disease, COVID-19 first causes lung diseases. Among the 238 anti-COVID-19 CHMs selected in our study, 42.02\% of them possess the character of meridian tropism in Fei. Besides, among the $238 \mathrm{CHMs}$, heat-clearing herbs $(29.41 \%)$ accounted for the largest proportion, followed by tonic herbs $(9.66 \%)$. It is suggested that CM may treat COVID-19 by clearing heat (resisting virus and inflammation) and tonifying deficiency (immunomodulation). The pathological study of COVID-19 also confirmed that the pathological changes were mainly caused by damage to the lung and immune system. Due to varied foundation diseases, other viscera suffered differently, mostly secondary damage. ${ }^{(1)}$

It is also described in WU Ju-tong's "Wen Bing Tiao Bian" that " Warm diseases include Feng Wen, Wen Re, Wen Nue, Wen Du, Shu Wen, Shi Wen, Qiu Zao, Dong Wen and Wen Yi." A study by $\mathrm{Yu}$, et $\mathrm{al}^{(8)}$ showed that in patients in China, the main affected organ of COVID-19 infections was the Fei, and the etiology and mechanism of COVID-19 in CM was mainly due to "dampness". Ran, et $\mathrm{al}^{(9)}$ found that the disease pattern of $30.14 \%$ COVID-19 patients in Chongqing are pathogenic-heat obstructing in Fei. Among the 238 potential CHMs, heat-clearing herbs accounted for the largest proportion (29.41\%), and the drug pair of Forsythiae Fructus-Lonicerae Japonicae Flos is the most widely distributed active ingredient, and is selected as the predicted core drug 
pair in this study. The pair of Forsythiae FructusLonicerae Japonicae Flos is a treatment method suitable for the early stage of COVID-19 when the disease pattern is heat. We suggest that the COVID-19 patients should be treated by integrated $\mathrm{CM}$ and Western medicine as soon as possible, and the pair of Forsythiae Fructus-Lonicerae Japonicae Flos should be adopted at the early stage of COVID-19 when the disease pattern is heat. In this way the transmission of Sanjiao and Wei-Qi-YingXue in CM can be prevented, and the possibility of the disease aggravation may be reduced.

The drug pair of Forsythiae Fructus-Lonicerae Japonicae Flos has 121 targets for viral pneumonia. And 14 pathways are coincided with viral pneumonia. One study showed that the pair of Forsythiae Fructus-Lonicerae Japonicae Flos had antipyretic, anti-inflammatory, anti-free radical damage and immune-enhancing effects in fever rats, and the combined use of the two herbs was better than a single use. ${ }^{(10)}$ The antiviral, antibacterial, antipyretic and anti-inflammatory effects of this drug pair were also revealed by a study by Ding, et al. ${ }^{(11)}$

TCR is a receptor on the surface of T cells and plays a key role in the function of $T$ cells and the formation of immune synapses. It provides the connection between $T$ cells and antigen presenting cells. TCR can specifically recognize peptides, and transform extracellular recognition into signals that can be transmitted to the interior of the cell. It can promote many signal cascade reactions by inducing tyrosine kinase activation and activating downstream mitogen-activated protein kinases, protein kinase C, calcium and other signal pathways, and thereby finally achieve T cell activation. ${ }^{(12)}$ One study showed that the extract of Flos Lonicerae had an immunosuppressive effect, and was capable of significantly inhibiting the phagocytosis of mononuclear macrophages in mice and suppressing various inflammatory reactions in the early, middle and late stages of inflammation. ${ }^{(13)}$

In this, we found that the pathways enriched with more than 4 targets were pertussis, tuberculosis, TCR and the TNF signaling pathways in the order of $P$ value from small to large in the drug pair of Forsythiae Fructus-Lonicerae Japonicae Flos. Therefore, this drug pair may play anti-inflammatory and immunomodulatory roles in COVID-19 through these signaling pathways, demonstrating the multitarget, multi-channel and multi-effect features of $\mathrm{CHM}$. The results of this study provided the basis for further studies on the molecular mechanism of the drug pair of Forsythiae Fructus-Lonicerae Japonicae Flos in the treatment of COVID-19.

In this study, the molecular compounds of $\mathrm{CHM}$ were mined from the TCMSP database based on virtual screening via molecular docking technology and network pharmacology, with SARS-CoV-2 3CL hydrolytic enzyme and ACE2 as receptors, and a reasonable virus inhibition scheme of $\mathrm{CHMs}$ was put forward. The results fully reflected the complexity of the mechanism of action of multi-component and multi-target $\mathrm{CHM}$, as well as the versatility and extensiveness of the pharmacological action of CHM. The method proposed in this study can provide reference for the development of effective combinations of CHMs for treating COVID-19. In view of the limitations of the virtual screening results, further in vitro and in vivo experiments are needed to verify the results of this study, so as to provide the experimental basis for the development of natural antiviral drugs.

\section{Conflict of interest}

The authors declare that they have no conflict of interests.

\section{Author Contributions}

All authors participated in the review of the manuscript. Gao LQ, Xu J and Chen SD analyzed data and wrote the manuscript.

\section{REFERENCES}

1. National Health Commission of the People's Republic of China, National Administration of Traditional Chinese Medicine. Diagnosis and treatment plan for pneumonia caused by novel coronavirus infection (3th to 7th ed). Available at http://www.nhc.gov.cn/yzygj/s7653p/new_list.shtml.

2. Chen JY, Shi JS, Yau TO, Liu C, Li X, Zhao Q, et al. Bioinformatics analysis of the 2019 novel coronavirus genome. Chin J Biol (Chin):1-10[2020-03-13]. Available at http://kns. cnki.net/kcms/detail/23.1513.q.20200120.0839.002.html.

3. Xu XT, Chen P, Wang JF, Feng JN, Zhou H, Li X, et al. Evolution of the novel coronavirus from the ongoing Wuhan outbreak and modeling of its spike protein for risk of human transmission. Sci China (Life Sci) 2020;63:457-460.

4. Zong $\mathrm{Y}$, Ding ML, J KK, Ma ST, Ju WZ. Exploring active compounds of Da-Yuan-Yin in treatment of COVID-19 
based on network pharmacology and molecular docking method. Chin Tradit Herb Drugs (Chin) 2020;51:836-844.

5. Wang W, Wang YW, Ma S, Li RF. Analysis on strategy, participation rate and cure effect of traditional Chinese medicine in treating COVID-19 in 23 provinces (Municipalities and Autonomous Regions). World J Tradit Chin Med (Chin) 2020;15:813-818.

6. Hirayama N. Docking simulations between drugs and HLA molecules associated with idiosyncratic drug toxicity. Drug Metab Pharmacokinet 2017;32:31-39.

7. Li J, Zhao P, Li Y, Tian Y, Wang Y. Systems pharmacologybased dissection of mechanisms of Chinese medicinal formula Bufei Yishen as an effective treatment for chronic obstructive pulmonary disease. Sci Rep 2015;5:15290.

8. Yu MK, Chai QY, Liang CH, Ding YQ, Lin ZY, Gao JQ, et al. An analyze of the traditional Chinese medicine prevention and treatment interventions for COVID-19. Chin Tradit Herb Drugs (Chin) 2020;61:383-387.

9. Ran J, Li YP, Li QT, Liu HB, Zeng CF, Ren Y, et al. Study of TCM syndrome in 209 novel coronavirus pneumonia cases of Chongqing in 2020. J Emerg Tradit Chin Med (Chin):1-3[2020-03-13]. Available at http://kns.cnki.net/ $\mathrm{kcms} /$ detail/50.1102.R.20200305.1611.002.html.

10. Duan HY, Ma C. Experimental study on pyretolysis mechanism of compatible application of Japanese Honeysuckle Flower Bud and Weeping Forsythia Fruit. J Modern J Integr Tradit Chin West Med (Chin) 2009;18:1214-1216.

11. Ding $X Y$, Lin $Z J$, Wang $D$. Research progress on constituents and pharmacological actions of Lonicerae Japonicae Flos, Forsythiae Fructus and their combination. Shandong Sci (Chin) 2019;32:36-41.

12. Liang JJ, LV J, Lu LR. The regulation and function of TCR signaling pathway. Chin Bull Life Sci (Chin) 2016;28:153-161.

13. Cui $X Y$. The antiinflammatory and immunomodulation effects of the extract of Louicera Japonica Thunb. Chin Pharm (Chin) 2011;20:8-9.

(Accepted April 23, 2020) Edited by WANG Wei-xia 